\title{
On the Stability of Dilatant Hardening for Saturated Rock Masses
}

\author{
JAMES R. RICE \\ Division of Engineering, Brown University, Providence, Rhode Island 02912
}

\begin{abstract}
Fissured rock masses tend to dilate as they are deformed inelastically toward failure. When the rock is fluid saturated and the time scale does not allow drainage, suctions are induced in the pore fluid, and by the effective stress principle the rock is dilatantly hardened over the resistance that it would show to a corresponding increment of drained deformation. This paper considers a compressed layer of saturated rock deformed in shear. Inelastic stress-strain relations are formulated, and these relations illustrate dilatant hardening when the layer is sheared without drainage at its boundaries. Parameters governing the slope of the hardened stress-strain curve are identified; these parameters predict a rising slope even when that presumed for homogeneous deformation under drained conditions is unstably falling. However, it is shown that the amount of dilatant strengthening that can actually be realized is limited by an instability. In particular, when the corresponding drained stress-strain curve has become unstable, small nonuniformities in the deformation field are shown to grow by local diffusive fluxes of pore fuid, and this is taken as the inception of localized shearing in a fault zone.
\end{abstract}

Fissured rock masses are observed to dilate as they are deformed inelastically toward failure under confining pressure [e.g., Brace et al., 1966]. This arises from frictional sliding on microcracks, which props them open at asperities and initiates further local tensile fissuring at their tips. The phenomenon in groundwater-saturated rock has received intensive recent interest $[$ Nur, 1972; Scholz et al., 1973; Whitcomb et al., 1973] due to its possible association with seismically observable precursors to shallow-focus earthquakes. When rock is saturated and the time scale of deformation is too short for diffusional drainage, the tendency to dilate will induce suction in the pore fluid. This suction augments the effective normal stress on fissure surfaces and hence dilatantly hardens the rock against continuing deformation. The phenomenon was first discussed for granular materials by Reynolds and is well known in the soil mechanics literature. It has been studied in connection with seismic sources by Frank [1965] and observed by Brace and Martin [1968] in triaxial tests of saturated rock. Indeed, the possibility arises that rock in shallow-focus fault regions is stabilized by its pore fluid against rapid failure during the premonitory period, when large-scale dilatancy seems to be taking place.

Hence the processes and the extent of dilatant strengthening of saturated rock are of great interest, as are also the conditions for its terminal loss of stability to localized deformation in a narrow fault zone. Frank [1965] has addressed the instability conditions for a special model of dilatant granular material. The present paper focuses attention on a compressed layer of saturated rock that is sheared toward failure. Dilatant inelastic constitutive relations are formulated that incorporate the Terzaghi-Biot effective stress principle and Darcy diffusion in the layer. In this way the parameters governing dilatant hardening are established, and conditions are derived for its loss of stability.

\section{Formulation of Stress-STrain and Diffusion RELATIONS}

We consider a layer of saturated, fissured rock that is sheared in plane strain (Figure 1) in such a way that there is no extensional strain in the $x$ direction and displacements $u_{x}, u_{y}$ of material points vary only with $y$ (and time $t$ ). The nonzero

Copyright $(1975$ by the American Geophysical Union, strain components in these circumstances are the shear $\gamma$ and the dilation $\epsilon$, where

$$
\gamma(y, t)=\frac{\partial u_{x}(y, t)}{\partial y} \quad \epsilon(y, t)=\frac{\partial u_{y}(y, t)}{\partial y}
$$

The stresses conjugate to these deformations are $\tau$ and $-\sigma(\sigma$ is positive if it is compressive) as illustrated; additional reaction stresses act as is necessary to maintain the constraints of nonextension in the $x$ direction and in that perpendicular to the $x$, y plane.

The layer is sheared into an inelastically deformed state, and incremental stress-strain relations must be written that include the possibility of both elastic unloading and further plastic loading. Here 'plastic' is intended to denote inelastic or irrecoverable deformation of any kind; of course, in the present case of brittle rock the primary plastic mechanisms will involve frictional sliding on fissures and local tensile cracking from their tips. In order to describe the elastic response elicited upon unloading the layer it is simplest to adopt the effective stress principle as it is normally used in soil mechanics and thus to assume that for elastic unloading,

$$
d \gamma=(1 / G) d \tau \quad d \epsilon=-(1 / M) d(\sigma-p)
$$

where $p$ is the pore fluid pressure and $G$ and $M$ are the incremental elastic unloading moduli in shear and uniaxial strain (e.g., Figure $2 a$ ). These moduli may in general vary during the deformation. Equation (2) implies that the pore fluid and mineral constituents of the fissured rock undergo negligible change in density, so that all dilation $\epsilon$ is explainable in terms of the volume of fluid either drawn into $(d \varepsilon>0)$ or squeezed out of $(d \epsilon<0)$ a material element. This is not often a good approximation for rock, and a more elaborate treatment taking account of the compressibility of the constituents is given in the appendix. When the increment of deformation is elastic plastic, plastic strain increments $d^{p} \gamma, d^{p} \epsilon$ are added to those of (2):

$d \gamma=\frac{1}{G} d \tau+d^{p} \gamma \quad d \epsilon=-\frac{1}{M} d(\sigma-p)+d^{p} \epsilon$

Note that $d^{p} \gamma, d^{p} \varepsilon$ are plastic strain increments in the sense that if the increment of inelastic deformation were followed by an increment of elastic unloading, which restored $\tau, \sigma$, and $p$ to 


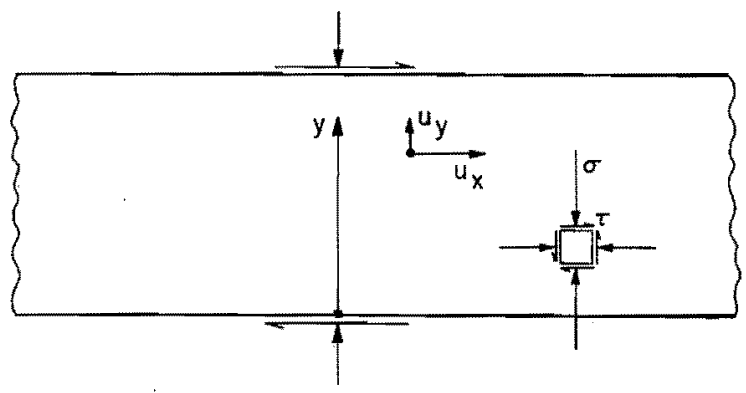

Fig. 1. Saturated rock layer, sheared in plane strain while it is subjected to compressive stress. Displacements vary spatially only with $y$.

their values before the inelastic increment, these would be the permanent strains accumulated in the infinitesimal load-unload cycle just described. It should be noted that this manner of defining plastic strain increments arises naturally in internal variable characterizations of inelastic behavior of solids [Rice, 1971]. Further, in the present case, for which plastic deformation (microfissuring) can alter elastic unloading moduli, there is an essential distinction between these plastic strain increments and corresponding increments of the strain that would remain upon complete unloading to zero stress.

To develop the form of the constitutive relation for plastic straining, consider first an increment of drained shear deformation in which $\sigma$ and $p$ are kept constant. We shall then write

$$
d^{p} \gamma=(1 / H) d \tau \quad \sigma, p=\text { const }
$$

where $H$ is the plastic hardening modulus. It is related to the instantaneous tangent modulus by $H_{\text {tan }}=H /(1+H / G)$ (Figure $2 a$ ) and is either positive or negative according to whether the $\tau$ versus $\gamma$ curve is rising (hardening) or falling (softening), although the falling portion may never be observed as homogeneous deformation in a drained test. For more general elastic-plastic deformations, in which $\sigma$ and $p$ do not remain constant, we utilize the principle of effective stress and write

$$
d^{p} \gamma=(1 / H)[d \tau-\mu d(\sigma-p)]
$$

where $\mu$ is a macroscopic friction parameter representing the effect of effective normal stresses in inhibiting sliding on microfissure surfaces. It is the local slope of the "yield surface" (separating elastic and inelastic states) in the plane of $\tau$ and ( $\sigma$ - $p$ ) (Figure 2b).

In general, sliding on a nonplanar fissure induces a certain amount of dilatancy by the propping open of the fissure and often by further cracking at an angle with the fissure due to local tensile stresses along its periphery. Thus it appears plausi-

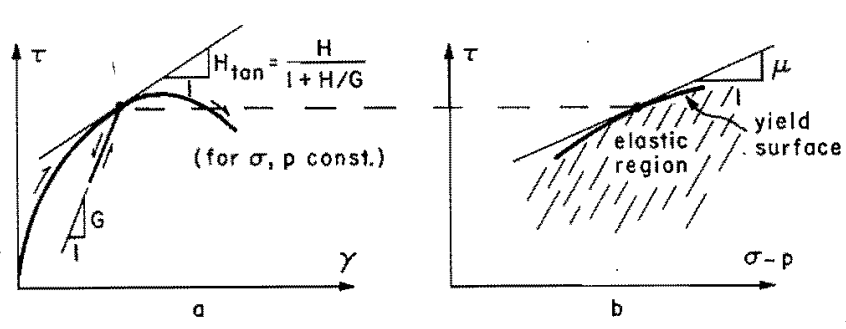

Fig. 2. Stress-strain data for saturated rock. (a) Shear stress-strain relation under drained conditions, $\sigma$ and $p$ being constant. (b) Effect of effective compressive stress on shear stress required for continuing inelastic deformation. ble to assume that the increment of plastic dilation $d^{p} \epsilon$ bears some fixed ratio to $d^{p} \gamma$ during a plastic increment regardless of the ratio of $d \tau$ to $d(\sigma-p)$ (so long as this ratio does indeed correspond to plastic response). We let $\beta$ be the dilatancy ratio:

$$
d^{p} \varepsilon / d^{p} \gamma=\beta
$$

where $\beta$ will vary with the amount of deformation. It can be assumed always to be positive in a program of sustained deformation for an initially coherent rock sample. Indeed, average values of the order of $\beta \approx 0.3$ are consistent with the results of Brace et al. [1966] on dry rock samples. Their Figure 6 suggests that $\beta$ may increase by perhaps a factor of 2 in going from the inception of fissuring to conditions very near failure. Also, their results show that $\beta$ will decrease with increasing confining pressure, which should be interpreted as meaning that $\beta$ will decrease with increasing effective stress $\sigma-p$ in the present fluid-saturated case. By contrast, loosely packed granular materials, such as sands, tend to go to a more dense state as a result of shear, and in such cases, $\beta$ is negative. With this interpretation the present framework could be applied also to liquefaction of loose, saturated sand deposits, although the topic is not pursued here. Hence with (3), (5), and (6) the complete stress-strain relation for elastic-plastic load increments is

$d \gamma=(1 / G) d \tau+(1 / H)[d \tau-\mu d(\sigma-p)]$

$d \epsilon=-(1 / M) d(\sigma-p)+(\beta / H)[d r-\mu d(\sigma-p)]$

Nonuniformities of pore pressure create diffusive flows in the layer, and these flows are assumed to take the Darcy form:

$$
q=-\rho \kappa \partial p / \partial y
$$

where $q$ is the fluid mass crossing unit area per unit time in the $y$ direction, $\rho$ is the mass density of homogeneous pore fluid at the pressure $p$ (and at the prevailing temperature), and $k$ is the permeability. For the present model with an incompressible fluid constituent, $\rho=$ const, and the equation of mass conservation coincides with that of volume conservation: the divergence of the apparent fluid velocity $q / \rho$ at a point is balanced by the rate of increase of volumetric strain there:

$$
\frac{\partial(q / p)}{\partial y}+\frac{\partial \epsilon}{\partial t}=0
$$

By using Darcy's law, (9) becomes

$$
\partial / \partial y[\kappa \partial p / \partial y]=\partial \epsilon / \partial t
$$

The only remaining physical requirement is that of continuing stress field equilibrium during the quasi-static deformation; when the one-dimensional spatial variation is given and body forces are neglected, the equilibrium equations reduce to

$$
\partial \tau / \partial y=0 \quad \partial \sigma / \partial y=0
$$

so that the stresses must be spatially uniform and vary only with time during the deformation.

The foregoing description has been limited to the case of unidirectional shearing to exhibit most simply in the following sections the dilatant hardening phenomenon and the conditions for its loss of stability. However, dilational stress-strain relations of a similar elastic-plastic construction have been proposed for arbitrary deformation states by $R$ udnicki and Rice [1974], who use them as a basis for establishing material instability conditions at which previously homogeneous deformations can localize into a narrow shear band. In these, as 
is true in the present work, an increment of shear stress is assumed to cause dilation only via the plastic deformation mechanism, $d^{p} \epsilon$ accompanies $d^{p} \gamma$, and plasticity is induced by stress states meeting the current yield criterion. A different approach is advocated by Stuart and Dieterich [1974], who consider isotropic materials for which the state of strain is a function only of the state of stress (i.e., isotropic elastic materials or initially isotropic inelastic materials that are considered only for special classes of monotonic deformation in which, for example, all stress components increase in fixed proportion to one another), and remark that as a consequence of the isotropic tensor representation theorem an amount of dilation is induced that is proportional to the square of the shear stress to the lowest nonzero order in a series expansion. Of course, the dilational effect during actual elastic response is small. Also, this type of representation cannot be adopted for strongly nonproportional stress variations in the inelastic range; e.g., a distinction between elastic unloading and further inelastic loading cannot be made. It may be noted in addition that if the inelasticity sets in only above some finite yield stress, a truncated series expansion about an unstressed state will not be suitable and will have to be replaced by a more elaborate functional form.

\section{Homogeneous Deformation With Dilatant HaRdening}

Suppose that drainage is prohibited at the boundaries of the layer and that physical properties are uniform within it. Then when the layer boundaries are displaced in shear, a possible solution entails homogeneous deformation throughout it. We shall later examine the stability of this solution. Evidently, $q=$ 0 for all $y$, and by (9) this requires that $\partial \epsilon / \partial t=0$ or $\epsilon=0$ throughout the deformation; since $d \epsilon=0$, one writes from the second equation of $(7)$ that

$$
\begin{gathered}
0=-\frac{1}{M} d(\sigma-p)+\frac{\beta}{H}[d \tau-\mu d(\sigma-p)] \text { or } \\
d(\sigma-p)=[\beta M /(H+\mu \beta M)] d \tau
\end{gathered}
$$

which shows the effective stress increment induced in an undrained increment of shear. When $\sigma$ is constant, $d(\sigma-p)$ is the increment of suction induced in the pore fluid. Substituting this last result into the first equation of (7) yields

$$
\begin{gathered}
d \gamma=\left[\frac{1}{G}+\frac{1}{H}\left(1-\frac{\mu \beta M}{H+\mu \beta M}\right)\right] d \tau \quad \text { or } \\
d \tau=\frac{H+\mu \beta M}{1+(H+\mu \beta M) / G} d \gamma
\end{gathered}
$$

This equation shows the dilatant hardening effect; the corresponding equation when the layer is drained at its boundaries and sheared under constant $\sigma$ at a sufficiently slow rate that no pore suctions develop, is (from the first equation of (7))

$$
d \tau=\frac{H}{1+H / G} d \gamma
$$

Hence the plastic hardening modulus $H$ is augmented to $H+$ $\mu \beta M$ (Figure 3 ) during undrained deformation. The increase in hardening modulus is thus the product of the elastic compressibility madulus $M$ with the friction coefficient $\mu$ and the dilatancy ratio $\beta=d^{p} \epsilon / d^{p} \gamma$. If $\mu \beta M$ is sufficiently great, the dilatantly hardened stress-strain curve will continue to rise, as is illustrated, even after $H$ has turned negative and the curve for drained deformation is descending.
It thus appears that very great increases in strength can be attained by dilatant hardening, especially when it is given that $\mu$ and $\beta$ may be assumed to have values typically in the ranges $0.5-1.0$ and $0.2-0.4$, respectively. These values suggest an augmentation of $H$ by $0.1-0.4 M$, which can mean substantial strengthening. Indeed, significant strength increases are observed in undrained tests [Brace and Martin, 1968]. However, the steadily rising dashed curve shown in. Figure 3 cannot be realized in practice, because homogeneous dilatantly hardened deformation becomes unstable.

\section{INSTABILITY}

Consider again the shearing of the layer while drainage is prevented at its boundaries, but let all the pertinent variables be written in the form

$$
\begin{gathered}
\gamma=\gamma_{0}+\tilde{\gamma} \quad \epsilon=\epsilon_{0}+\bar{\epsilon} \quad \tau=\tau_{0}+\tilde{\tau} \\
\sigma=\sigma_{0}+\tilde{\sigma} \quad p=p_{0}+\tilde{p}
\end{gathered}
$$

where the quantities $\gamma_{0}, \tau_{0}$, etc., represent the solution of the last section for homogeneous deformation and where $\tilde{\gamma}, \tilde{\tau}$, etc., are perturbation quantities. Specifically, the layer is sheared by application of a monotonically increasing shear stress $\tau_{0}=$ $\tau_{0}(t)$ to its boundary while the normal stress is held constant at $\sigma_{0}$. By (11) the stress field is uniform within the layer, and thus $\tilde{\tau}=\tilde{\sigma}=0$. Also, the homogeneous solution involves no volume change, and so $\epsilon_{0}=0$. Thus

$$
\begin{array}{cc}
\gamma=\gamma_{0}+\tilde{\gamma} & \epsilon=\tilde{\epsilon} \quad \tau=\tau_{0} \\
\sigma=\sigma_{0} & p=p_{0}+\tilde{p}
\end{array}
$$

Further, from the second equation of (12) together with the fact that $\sigma_{0}=$ const and from the second equation of (13) for the homogeneous solution, $p_{0}$ and $\gamma_{0}$ satisfy

$$
\begin{aligned}
& d p_{0}=-\frac{\beta M}{H+\mu \beta M} d \tau_{0} \\
& d \gamma_{0}=\frac{1+(H+\mu \beta M) / G}{H+\mu \beta M} d \tau_{0}
\end{aligned}
$$

where the various constitutive parameters $G, M, H, \mu$, and $\beta$ are evaluated at the deformation state achieved in the homogeneous solution.

It is simplest to form the equations for the perturbation field by neglecting the effect of the perturbations on these constitutive parameters, and this is of no detriment to the end result. It is equivalent to the assumption that the parameters are lo-

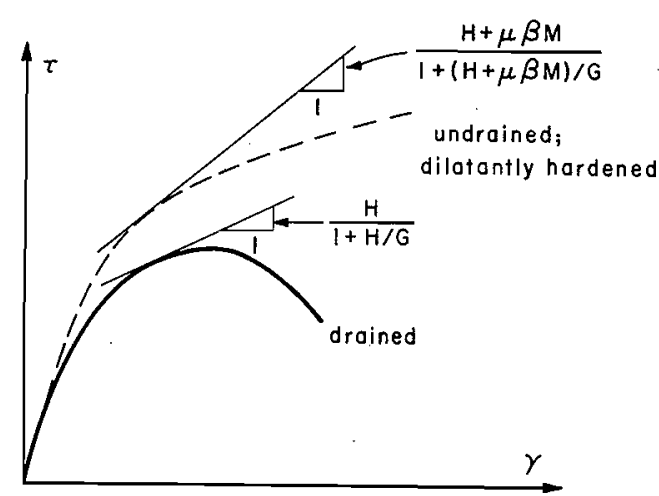

Fig. 3. Comparison of stress-strain curves for drained and undrained homogeneous deformation: $H \rightarrow H+\mu \beta M$. Undrained, dilatantly hardened deformation becomes unstable when $H<0$. 
cally constant over the range of deformations for which the evolution of the perturbations is considered. Since (7) and (10) are linear in the field parameters and are satisfied exactly by the homogeneous field, subtraction from them of the homogeneous forms of (7) and (10) leaves the same equations in terms of the perturbation parameters. Thus from (7)

$d \tilde{\gamma}=\frac{\mu}{H} d \tilde{p} \quad d \tilde{\epsilon}=\frac{1}{M} d \tilde{p}+\frac{\beta \mu}{H} d \tilde{p}=\frac{H+\mu \beta M}{M H} d \tilde{p}$

or when it is realized that these refer to increments of deformation at a fixed material point,

$$
\frac{\partial \tilde{\gamma}}{\partial t}=\frac{\mu}{H} \frac{\partial \tilde{p}}{\partial t} \quad \frac{\partial \tilde{\epsilon}}{\partial t}=\frac{H+\mu \beta M}{M H} \frac{\partial \tilde{p}}{\partial t}
$$

Also, from (10),

$$
\kappa \partial^{2} \tilde{p} / \partial y^{2}=\partial \tilde{\epsilon} / \partial t
$$

By substituting from the second equation of (19) it is thus seen that perturbations in pore pressure satisfy

$$
\frac{\partial^{2} \tilde{p}}{\partial y^{2}}=\frac{1}{c} \frac{\partial \tilde{p}}{\partial t} \quad c=\frac{\kappa M H}{H+\mu \beta M}
$$

Further, from the first equation of (19), nonuniformities $\tilde{\gamma}$ in the amount of shear will either grow or dissipate in accord with those of pore pressure. Equation (21) is the ordinary diffusion equation, and thus the deformation is stable, in that pressure nonuniformities dissipate, so long as $c>0$. But when $c<0$, this is equivalent to a diffusion equation for which time runs backward, and any distribution of pressure nonuniformities, except those devoid of short-wavelength components, grows immediately into a singular distribution.

The permeability $\kappa$ and elastic compressibility $M$ are always positive. Further, for the range of interest, in which dilatant hardening is exhibited in homogeneous deformation (Figure 3), $H+\mu \beta M>0$. Hence the sign of $c$ is the same as that of $H$, namely, that of the modulus for an inelastic increment of deformation under drained conditions. Thus dilatant hardening is stable only in those circumstances for which the underlying drained deformation would be stable: $H>0$.

The smallest Fourier wavelengths of the initial nonuniformity grow most rapidly. In particular, solutions to (21) of the kind

$$
\tilde{p}=P(t) \cos (2 \pi y / \lambda)
$$

are readily found where, $P(0)$ is the initial amplitude of the nonuniformities of wavelength $\lambda$,

$$
P(t)=P(0) e^{r t} \quad r=-4 \pi^{2} c / \lambda^{2}
$$

Hence the perturbations grow whenever $c<0$ (i.e., $H<0$ ), and those of the shortest wavelength grow most rapidly. Solutions of this kind are compatible with zero flow through the boundaries of the layer whenever $n \lambda / 2$ is equal to the layer thickness where $n$ is a positive integer. The corresponding perturbation in the shearing rate is, from the first equation of (19),

$$
\frac{\partial \tilde{\gamma}}{\partial t}=-\frac{4 \pi^{4} \kappa}{\lambda^{2}} \frac{\mu M}{H+\mu \beta M} P(0) e^{r t} \cos (2 \pi y / \lambda)
$$

and if only a single wavelength is present, zones of local elastic unloading, interspersed with zones of intensified shear, will set in when the amplitude of this rate equals that of the homogeneous solution:

$$
\frac{d \gamma_{0}}{d t}=\frac{1+(H+\mu \beta M) / G}{H+\mu \beta M} \frac{d \tau_{0}}{d t}
$$

from the second equation of (17), on which it is superposed.

A more general perturbation analysis would consider the various constitutive parameters to vary with $\gamma$ and $\epsilon$, so that these are of the form

$$
H=H\left(\epsilon_{0}, \gamma_{0}\right)+\tilde{\epsilon} \frac{\partial H}{\partial \epsilon}\left(\epsilon_{0}, \gamma_{0}\right)+\tilde{\gamma} \frac{\partial H}{\partial \gamma}\left(\epsilon_{0}, \gamma_{0}\right)
$$

Also, if initial small nonuniformities of properties were present in the layer, there would be an additional small term dependent explicitly on $y$. The effect of this is to add terms to (19) that are linear in the perturbation amplitudes, each of the added terms being multiplied by $d \tau_{0} / d t$. However, the solution for growth of perturbations in the short-wavelength limit is governed only by the highest-order spatial and time derivatives of the differential equations, and in this respect the conclusions derived above on instability are unaltered; the growth equations for finite wavelengths become yery much more complicated.

The instability condition $H<0$ is the same as that derived by Frank [1966], when his special granular constitutive relation is rewritten in the present notation. Frank's assumptions are equivalent to setting $G=M=\infty$, so that only plastic strain occurs, and to requiring that $\tau=($ const $+\beta)(\sigma-p)$ for deformation, where $\beta$ is considered to be a function of $\gamma$ and has the same interpretation as it does in (6). Rewriting this in the form of (5), we see that $\mu=$ (const $+\beta$ ) and $H=(\sigma-p)$ $d \beta / d \gamma$. Thus the instability condition $H<0$ is equivalent to $d \beta / d \gamma=d^{2} \varepsilon / d \gamma^{2}<0$, as given by Frank for his granular model.

It should also be remarked that the underlying drained deformation will first become unstable at a point corresponding to a maximum in the stress-strain curve (i.e., $H=0$ ) only under certain special deformation states, in particular for the kind of deformation state considered in Figure 1, for which a nondeforming plane (the $x z$ plane) exists. Indeed, Frank [1966] finds for his simple rigid-plastic model that instability can occur only if such a nondeforming plane exists. On the other hand, the more elaborate elastic-plastic analysis of Rudnicki and Rice [1974] confirms that instability, in the form of deformation localization into a shear band, can occur for more general deformation states, and depending on the particular state, it may set in while the stress-strain curve is either slightly rising or slightly falling. However, such predictions are found to be very sensitive to the exact manner in which the constitutive response is modeled for abrupt changes in the pattern of straining.

\section{Concluding Discussion}

The analysis has demonstrated the dilatant hardening phenomenon in undrained deformation and has shown that it becomes unstable when the underlying drained behavior of the rock is unstable. It was shown that if $H$ is the incremental plastic shear modulus for drained deformation, then $H+\mu \beta M$ is the corresponding modulus for undrained deformation (Figure 3), where $\mu$ is the friction coefficient, arising in the expression $d \tau-\mu d(\sigma-p)$ for the part of a shear stress increment that causes inelastic response; $\beta$ is the ratio $d^{p} \epsilon / d^{p} \gamma$ of an increment of inelastic dilation to an increment of shear; and $M$ is the elastic compression modulus of the layer as given by $d \epsilon=-d \sigma / M$ for drained elastic response. It is shown in the 
appendix that when the pore fluid and mineral constituents are compressible, the plastic shear modulus is $H+\mu \beta M^{\prime}$ for undrained deformation, where $M^{\prime}$ is given by (AI2) and (A13); $M^{\prime}$ can be significantly smaller than $M$ whenever $M v / K_{f}$ is not small in comparison with unity ( $v$ is the volume fraction of pores, and $K$, the fluid bulk modulus).

The results given show the way by which induced pore suctions can stabilize a saturated rock mass against failure and, by the instability analysis, show that the amount of dilatant strengthening attainable in this way is limited by conditions at which the underlying drained response becomes unstable. To model more closely dilatant strengthening as it might occur prior to an earthquake, it would seem of interest to study the shearing of a rock mass containing a weakened and heavily fissured zone that represents the effects of previous faulting. In contrast to the present analysis with impermeable boundaries the time scale of the deformation then becomes important. In deformations slow in comparison with diffusion times, all suctions within the weakened zone will be alleviated, and the strength that governs will be the drained strength of rock within that zone. But with more rapid deformations the zone should, at least initially, exhibit dilatant strengthening. This would force the abutting rock masses to carry a greater shear stress and hence could cause prefailure dilatancy to spread laterally from the fault zone. This is evidently necessary if the observed premonitory variation of stress wave travel times is to be attributed to dilatancy.

Time effects could also enter in the form of macroscopic rock creep, due to the growth of microfissures by stress corrosion cracking in the surrounding groundwater [Scholz, 1968]. Such crack growth under sustained shear stress would lead to overall dilatancy, and hence induced pore fluid suctions would again play a role in strengthening the rock or in slowing the rate of creep toward failure provided that the time scale is short in comparison with diffusion times.

\section{ApPendix: Formulation for Compressible Mineral ElÉments ANd Pore Flutd}

When the mineral elements and pore fluid within the layer are elastically compressible, it is necessary to generalize properly the elastic stress-strain relation (the second equation of (2)) for volumetric deformation and the mass conservation relations (equations (9) and (10)). It is assumed that the expressions (4)-(6) for inelastic portions of strain increments retain their validity in this case.

For simplicity the elastic formulation is given in terms of infinitesimal deformation, and an element of the rock skeleton having unit reference volume is considered. Hence at constant temperature the variation of free energy $\phi$ of this element, when it is saturated, during an elastic increment of deformation and/or matter addition is

$$
\delta \phi=\tau \delta \gamma-\sigma \delta \epsilon+\tilde{\mu} \delta m
$$

where $m$ is the mass of pore fluid contained within the unit reference volume and, following a recent presentation by Biot [1973], $\tilde{\mu}$ is its potential. Ignoring contributions to $\tilde{\mu}$ from differences in gravitational potential within the layer and computing the reversible work $\tilde{\mu} \delta m$ involved in transferring an incremental fluid mass to the element from a fluid reservoir at some fixed pressure (and the same temperature), while $\epsilon$ and $\gamma$ are held fixed, one readily computes that

$$
\tilde{\mu}=\int_{p_{\mathrm{o}}}^{p} \frac{d p^{\prime}}{\rho\left(p^{\prime}\right)}
$$

Here $p_{0}$ is the reservoir pressure, $p$ is the pressure that equilibrates the pore fluid against further exit from or entry into the element (i.e., the pore pressure), and $\rho=\rho(p)$ (the dependence on temperature not being explicitly noted) is the mass density of a homogeneous element of pore fluid at pressure $p$.

If we let $v=m / p$ be the apparent volume of pore fluid within the considered element of unit reference volume, so that $v$ is the apparent volume fraction of pores, then a Legendre transformation leads to

$$
\delta(\phi-\tau \gamma+\sigma \epsilon-\tilde{\mu} m)=-\gamma \delta \tau+\epsilon \delta \sigma-v \delta p
$$

for increments of $\tau, \sigma$, and $p$ that induce only elastic response. The right side of (A3) is an exact differential, and this implies the reciprocal relation [Biot, 1973]

$$
(\partial \epsilon / \partial p)_{\sigma_{\tau} \tau}=-(\partial v / \partial \sigma)_{p, \tau}
$$

For elastic deformation increments we write

$$
\begin{gathered}
d \gamma=\frac{1}{G} d \tau \quad d \epsilon=-\frac{1}{M} d(\sigma-p)-\frac{1}{M_{s}} d p \\
d v=-\frac{1}{N} d(\sigma-p)-\frac{v}{N_{s}} d p
\end{gathered}
$$

Here $M$ continues to be interpreted as the overall elastic compression modulus of the fissured mass due to an increment of $\sigma$ under drained conditions $(d p=0)$. It can be assumed that when $\sigma$ and $p$ are incremented by the same amount, $d \sigma=d p$, the increment $d \epsilon$ is governed almost entirely by the elastic moduli of the solid mineral elements within the layer, and hence $M_{s}$ is of the order of such moduli; it will be very much larger than $M$ for highly fissured rock. By similar reasoning, $N_{s}$ is of the same order, and $d p / N_{s}$ represents the fractional decrease of pore volume space when $d \sigma=d p$. The remaining coefficient $N$ is given by the reciprocal relation (A4), so that

$$
1 / N=(1 / M)-\left(1 / M_{s}\right)
$$

This completes the elastic description. During an inelastic deformation increment it is necessary to add plastic increments $d^{p} \gamma, d^{p} \epsilon$, and $d^{p} v$ to the terms of (A5). Since $d^{p} \epsilon$ reflects the dilatant increase in pore space due to the sliding-up of microcrack surfaces at asperities and further local tensile fissuring from their tips, it can be assumed that $d^{p} v=d^{p} \epsilon$. Hence by means of (5) and (6), equations (7) are generalized to

$$
\begin{aligned}
d \gamma=\frac{1}{G} d \tau+\frac{1}{H}[d \tau-\mu d(\sigma-p)] \\
d \epsilon=-\frac{1}{M} d(\sigma-p)-\frac{1}{M_{a}} d p \\
+\frac{\beta}{H}[d \tau-\mu d(\sigma-p)]
\end{aligned}
$$

$$
\begin{aligned}
d v=-\left(\frac{1}{M}-\frac{1}{M_{s}}\right) d(\sigma & -p)-\frac{v}{N_{s}} d p \\
& +\frac{\beta}{H}[d \tau-\mu d(\sigma-p)]
\end{aligned}
$$

where $\mu, \beta$, and $H$ have the same significance as earlier. The mass conservation equation $(9)$ is now generalized to

$$
\partial q / \partial y+\partial m / \partial t=0
$$

and when Darcy's relation (8) is used and $m=\rho v$ is rewritten, (10) is generalized to 


$$
\frac{\partial}{\partial y}\left(\rho \kappa \frac{\partial p}{\partial y}\right)=\frac{\rho v}{K_{t}} \frac{\partial p}{\partial t}+\rho \frac{\partial v}{\partial t}
$$

where $K_{t}=\rho d p / d p$ is the bulk modulus of the pore fluid.

The relations now established allow the generalization of the second equation of (13) for undrained homogeneous deformation and also the condition derived for its loss of stability to the case of compressible mineral elements and pore fluid. Indeed, for undrained homogeneous shear of the layer at constant $\sigma$, one sets $d m=0$ or

$$
d v=-\left(v / K_{f}\right) d p
$$

and uses this together with $d \sigma=0$ in the first and third equations of (A7) to derive

$$
\begin{aligned}
& d p=-\frac{\beta M^{\prime}}{H+\mu \beta M^{\prime}} d \tau \\
& d \tau=\frac{H+\mu \beta M^{\prime}}{1+\left(H+\mu \beta M^{\prime}\right) / G} d \gamma
\end{aligned}
$$

as the generalizations of the second equations of (12) and (13), where

$$
\frac{1}{M^{\prime}}=\frac{1}{M}+\frac{v}{K_{f}}-\frac{1}{M_{s}}-\frac{v}{N_{s}}
$$

Thus the previously given relations for undrained homogeneous deformation remain valid so long as $M$ is replaced by $M^{\prime}$, and the effect of the dilatant strengthening is to replace the plastic hardening modulus $H$ by $H+\mu \beta M^{\prime}$. The last two terms in the expression for $1 / M^{\prime}$ can be expected to be very much smaller than the sum of the first two, and thus

$$
M^{\prime} \approx \frac{M}{1+M v / K_{r}}
$$

It is therefore seen that compressibility of the constituents is of little effect when $M v / K_{f}$ is small in comparison with unity. On the other hand, when the pore volume fraction $\nu$ is large or when the prevailing temperature and the amount of induced suction in the pore fluid are such as to reduce $K_{f}$ to a small value, $M^{\prime}$ can be substantially less than $M$, and the slope of the dashed dilatantly hardened stress-strain curve of Figure 3 is greatly reduced.

The procedure followed earlier for small perturbations is readily generalized by adding $v=v_{0}+\tilde{v}$ to $(16)$, and the linearized relations (19) and (20) become

$$
\frac{\partial \tilde{\gamma}}{\partial t}=\frac{\mu}{H} \frac{\partial \tilde{p}}{\partial t} \quad \frac{\partial \tilde{\epsilon}}{\partial t}=\left(\frac{1}{M}-\frac{1}{M_{s}}+\frac{\mu \beta}{H}\right) \frac{\partial \tilde{p}}{\partial t}
$$

$$
\begin{gathered}
\frac{\partial \tilde{v}}{\partial t}=\left(\frac{1}{M}-\frac{1}{M_{s}}-\frac{v}{N_{s}}+\frac{\mu \beta}{H}\right) \frac{\partial \tilde{p}}{\partial t} \\
\kappa \frac{\partial^{2} \tilde{p}}{\partial y^{2}}=\frac{v}{K_{f}} \frac{\partial \tilde{D}}{\partial t}+\frac{\partial \tilde{v}}{\partial t}
\end{gathered}
$$

Hence analogously to (21), perturbations in pore pressure satisfy

$$
\frac{\partial^{2} \tilde{p}}{\partial y^{2}}=\frac{1}{c^{\prime}} \frac{\partial \tilde{p}}{\partial t} \quad c^{\prime}=\frac{\kappa M^{\prime} H}{H+\mu \beta M^{\prime}}
$$

The condition for unstable growth is that $c^{\prime}<0$, and the shortest-wavelength nonuniformities again grow most rapidly. Since $\kappa, \mu, \beta$, and $M^{\prime}$ are positive, the dilatantly hardened deformation becomes unstable when $H<0$, which is the same condition derived earlier that instability occurs when the underlying drained deformation becomes unstable.

Acknowledgments. This study was supported by the Advanced Research Projects Agency/National Science Foundation Materials Research Laboratory at Brown University and by NSF Geophysics Program grant GA-43380. The writer is grateful to J, W. Rudnicki for valuable discussions and to W. D. Stuart and N. Fujii for comments on the original manuscript.

\section{REFERENCES}

Biot, M. A., Nonlinear and semilinear rheology of porous solids, $J$. Geophys. Res., 78, 4924-4937, 1973.

Brace, W. F., and R. J. Martin, A test of the law of effective stress for crystalline rocks of low porosity, Int. J. Rock Mech. Miner. Sci., S, $415-436,1968$.

Brace, W. F., B. W. Paulding, Jr, and C. H. Scholz, Dilatancy in the fracture of crystalline rock, J. Geophys. Res., 71, 3939-3953, 1966.

Frank, F. C., On dilatancy in relation to seismic sources, Rev. Geophys. Space Phys., 3, 485-503, 1965.

Nur, A., Dilatancy, pore fluids, and premonitory variations of $t_{s} / t_{p}$ travel times, Bull. Seismol. Soc. Amer., 62, 1217-1222, 1972.

Rice, J. R., Inelastic constitutive relations for solids: An internal variable theory and its application to metal plasticity, J. Mech. Phys, Solids, 19, 433-455, 1971

Rudnicki, J. W., and J. R. Rice, Conditions for the localization of deformation in pressure-sensitive dilatant materials, Rep. NSF GA43380/2, Brown Univ., Providence, R. 1., 1974.

Scholz, C. H., Mechanism of creep in brittle rock, J. Geophys. Res., 73 , 3295-3307, 1968.

Scholz, C. H., L. R. Sykes, and Y. P. Aggarwal, Earthquake prediction: A physical basis, Science, 181, 803 810, 1973.

Stuart, W. D., and J. H. Dieterich, Continuum theory of rock dilatancy, paper presented at 3rd Congress, Int. Soc. Of Rock Mech., Denver, Colo., 1974.

Whitcomb, J. H., J. D. Garmany, and Y. P. Anderson, Earthquake prediction: Variation of seismic velocities before the San Fernando earthquake, Science, 180, 632-635, 1973.

(Received July 15, 1974; revised October 14, 1974; accepted October 19, 1974.) 